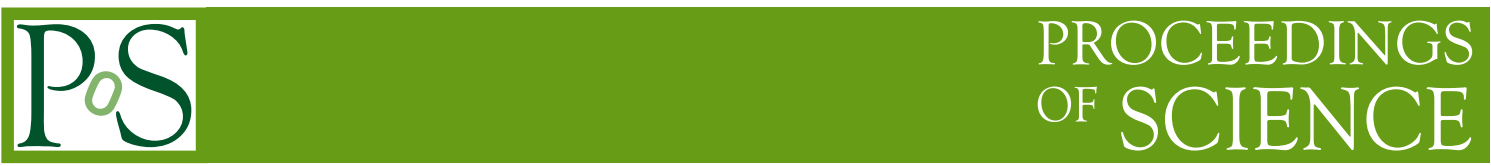

\title{
The CMS Level-1 muon triggers for the LHC Run II
}

\author{
Marcin Konecki* \\ University of Warsaw, Poland \\ E-mail: marcin.konecki@cern.ch
}

The CMS experiment implements a sophisticated two-level triggering system composed of Level-1, instrumented by custom-design hardware boards, and a software High-Level Trigger. A new Level-1 trigger architecture with improved performance is now being used to maintain high physics efficiency for the more challenging luminosity conditions experienced during Run II. The CMS muon detector was designed for preserving the complementarity and partially redundant muon detection systems, Cathode Strip Chambers (CSC), Drift Tubes (DT) and Resistive Plate Chambers (RPC), until they were combined at the input to the Global Trigger. The upgraded muon trigger combines information from the three muon detectors in the track reconstruction in order to obtain a better efficiency and lower rates. Advanced pattern recognition and MVA (Boosted Decision Tree) regression techniques implemented directly on the trigger boards allow high-momentum signal muons to be distinguished from the overwhelming low-momentum background. The algorithms for the selection of events with muons, both for precision measurements and for searches of new physics beyond the Standard Model, will be described in detail. The performance of the upgraded muon trigger system is evaluated, based on proton-proton collision data collected in Run II.

The 39th International Conference on High Energy Physics (ICHEP2018)

4-11 July, 2018

Seoul, Korea

${ }^{*}$ On behalf of the CMS Collaboration. 
For Run II, in 2015/16, following harsher LHC conditions, CMS has upgraded its entire Level-1 trigger [1]. In the legacy Run I trigger the information from three muon sub-detectors (DT, RPC,CSC) was processed independently by dedicated triggers and combined later. In the upgraded Run-II trigger the muon data from various types of detectors are combined already at muon candidate reconstruction step. The trigger reconstruction is worked out in Muon Track Finders (MTFs): barrel data are processed by BMTF, endcap by EMTF, while data from a barrel-endcap overlap region are treated by dedicated OMTF. Muon candidates reconstructed by MTFs are sent to the Global Muon Trigger (GMT) where duplicate candidates are eliminated.

The BMTF algorithm relies on hits grouped into "segment primitives" that provide muon position and incident angle in a barrel station. A segment is constructed from combined DT superlayers supplemented with RPC hits for better efficiency and bunch crossing determination. The muon track candidate finding is based on search for compatible segments in an extrapolation window between muon stations. The candidate kinematics is read from LUTs implemented in FPGA.

In the EMTF, pattern recognition selects CSC hits grouped into "stub primitives" that contribute to the muon track candidate. The candidate kinematics is assigned using large (GB) and fast memory indexed by stub positions. The memory-based $p_{T}$-LUTs are filled using results of the offline BDTs studies. In case of missing CSC information the RPC hits are used instead.

In the OMTF, data from DT, RPC and CSC detectors are treated equally. The algorithm compares actual hit pattern from all detectors with a limited number of "Golden Patterns". The Golden Pattern holds information about bending distribution in each layer for muons of a given $p_{T}$. The Golden Pattern best matched to a hit pattern in the event determines the candidate momentum.

The performance studies [2], see Fig. 1, show that more sophisticated online algorithms implemented in the upgraded MTFs have improved trigger performance compared to the legacy system in terms of reduced rate while preserving high efficiency of the trigger. The improved resolution and possibility of propagating of the measurements to the vertex allowed for exploiting complex algorithms, such as invariant mass triggers, that increase the physics potential of CMS.
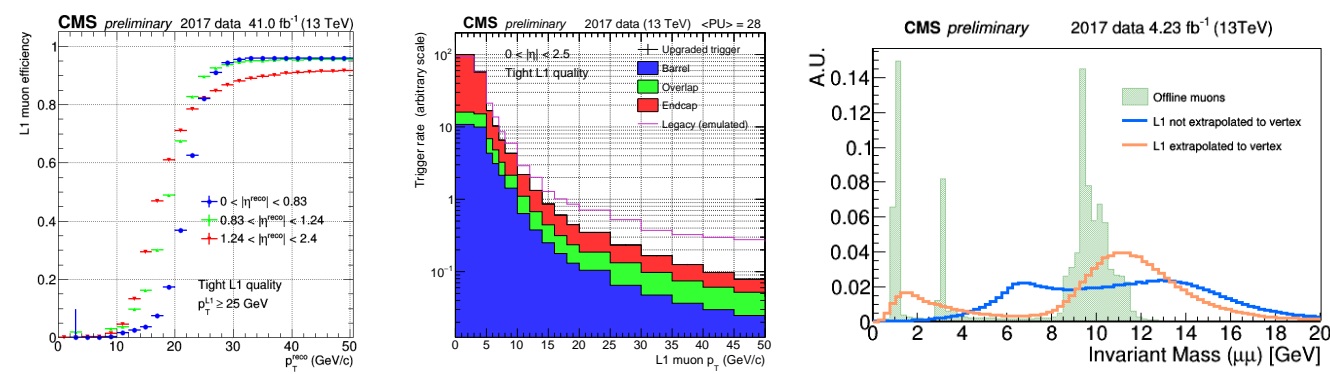

Figure 1: Level-1 muon trigger performance [2]: efficiency, rates and invariant mass distributions.

Acknowledgments: OMTF project supported by National Science Centre, Poland, grant UMO-2015/19/B/ST2/02861.

\section{References}

[1] CMS Coll., CMS Technical Design Report for the Level-1 Trigger Upgrade, CERN-LHCC-2013-011.

[2] CMS Coll., Performance Notes: CMS DP-2017/041, DP-2018/002, DP-2018/008, DP-2018/044. 